\title{
LIFETIME EXTENSION AND ROUTE STABILIZATION OF ENERGY-EFFICIENT BROADCAST ROUTING OVER MANET
}

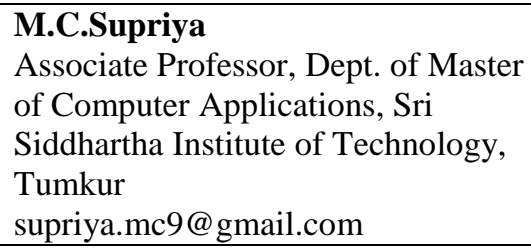

\author{
Dr. B.P.Mallikarjunaswamy \\ Professor, Dept. of Computer Science \\ and Engineering, Sri Siddhartha \\ Institute of Technology, Tumkur.
}

\author{
Rajani Narayan \\ Associate Professor, Dept. of Master \\ of Computer Applications, RNS \\ Institute of Technology, Bangalore.
}

\begin{abstract}
In this paper, we discuss energy efficient broadcast in ad hoc wireless networks. The problem of our concern is: Given an ad hoc wireless network, find a broadcast tree such that the energy cost of the broadcast tree is minimized. We address the problem of energy efficient multicast routing in wireless Mobile Adhoc Network (MANET). It is a challenging environment because every node operates on limited battery resource and multi-hop routing paths are used over constantly changing network environments due to node mobility. We define the network lifetime as duration of time until first node failure due to battery energy exhaustion and show that net-work lifetime for a multicast session can be significantly extended by additionally considering the residual battery energy as a parameter in cost metric functions for constructing a power efficient routing tree. Using simulation results, we show that the lifetime extension can lead to oscillatory behavior of routing path selection. We propose a solution to stabilize the oscillations by considering a statistical measure in our cost metric and present simulations that show the oscillation can be reduced greatly at a small cost of network lifetime. achieves a good performance in terms of lifetime by reducing energy consumption for communication and balancing the energy loads among all the nodes, which increases the network life.
\end{abstract}

\section{Keywords}

MANET, Adhoc Networks, Energy Efficient Routing, Multicasting Tree, BIP Algorithm

\section{INTRODUCTION}

Mobile ad hoc networks (MANETs) depict complex distributed systems that comprise wireless mobile nodes. These nodes can freely and dynamically self-organize into arbitrary and temporary ad hoc network topologies, thus allowing people and devices to seamlessly internet work in areas with no preexisting communication infrastructure, for example, disaster recovery environments [1].The routing protocols are used to route the packets from the source node to the destination. These routing protocols should be efficient in terms of quality of service (QoS) metrics and energy consumption to guarantee the data transmission over the wireless medium [2]. The internet engineering task force (IETF) has developed four routing protocols mainly for MANET [3]: ad hoc on demand vector: (AODV), dynamic source routing (DSR), topology dissemination based on reverse-path forwarding (TBRPF), and optimized link state routing (OLSR) [4].

The limited capacity of mobile nodes batteries represents a constraint for MANETs. Therefore, the design of energy efficient routes is a substantial need for saving energy and extending batteries and network lifetime. The failure of nodes caused by the battery exhaustion restricts the node's ability to transmit and receive or relay packets to others as an intermediate node. Thus, routing a packet between source destination pairs requires an adequate number of alive nodes with enough energy to forward the packets between them.

\section{BACKGROUND}

In this paper, the best effort to conserve energy at each instance of time and to extend 
the lifetime of the multicast session is made using a power efficient multicast tree and by updating this tree regularly in a strategic manner. We use a recently proposed powerefficient multicast tree construction algorithm as a building block and construct our cost functions. Based on these newly proposed cost functions, we derive solutions that lead to significant improvements in extending the network lifetime while reducing the oscillations in routing algorithms.

\subsection{Wireless Broadcast Advantage}

Fig.1(a) shows a single sender $\mathrm{S}$ with receivers $\mathrm{M}_{1}$ and $\mathrm{M}_{2}$ at distances of $\mathrm{d}_{1}$ and $\mathrm{d}_{2}$ respectively, from the sender. We assume that $\mathrm{d}_{2}>\mathrm{d}_{1}$ and the received power at a node varies as $\mathrm{d}_{\mathrm{i}}^{-\alpha}(\mathrm{i}=1,2)$ where $\alpha$ is the path loss factor satisfying $(2 \leq \alpha \leq 4)$. Hence, the transmission power required to reach a node at a distance $d_{i}$ is proportional to $\mathrm{d}_{\mathrm{i}}{ }^{\alpha}$ assuming the proportionality constant is 1 . Fig.1(b) shows the broadcast nature of the wireless medium for omni-directional antenna in which a unit of message sent to receiver A at the boundary of the circle reaches every node within the circle for "free." In order to transmit an identical message to nodes $\mathrm{M}_{1}$ and $\mathrm{M}_{2}, \mathrm{~S}$ can use two unicast transmissions with individual power $\mathrm{d}_{1}{ }^{\alpha}$ and $\mathrm{d}_{2}{ }^{\alpha}$ with total expenditure of $\left(\mathrm{d}_{1}{ }^{\alpha}+\mathrm{d}_{2}{ }^{\alpha}\right)$. However, it can be reduced to $\max \left(\mathrm{d}_{1}{ }^{\alpha}, \mathrm{d}_{2}{ }^{\alpha}\right)$ by taking advantage of the fact that the wireless medium is naturally "broadcast." Under this assumption, the sender has to choose between the following two strategies: (a) if $\mathrm{d}_{2}{ }^{\alpha}>\mathrm{d}_{1}{ }^{\alpha}+$ $\mathrm{d}_{12}{ }^{\alpha}$, transmit to $\mathrm{M}_{1}$ and let $\mathrm{M}_{1}$ transmit to $\mathrm{M}_{2}$, (b) otherwise, transmit to $\mathrm{M}_{2} \quad\left(\mathrm{M}_{1}\right.$ will automatically receive it due to wireless broadcast advantage since $d_{2}>d_{1}$ ). Hence, joint consideration of transmission and routing leads to savings in battery energy.

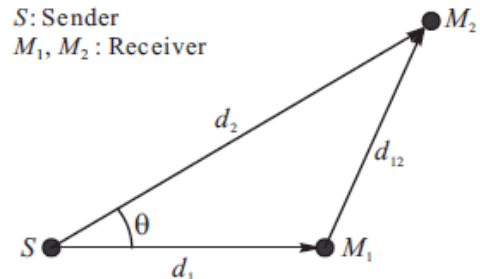

(a)

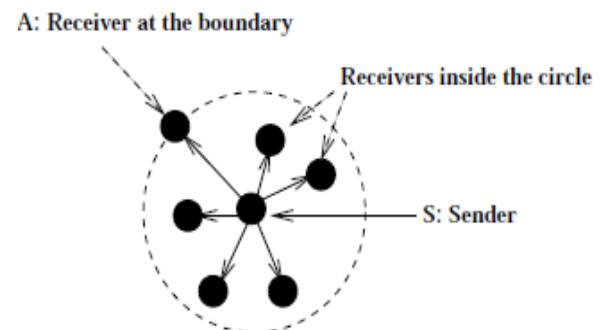

(b)

Figure 1: (a) Geometric construct of a sender $S$ and receivers $M 1$ and $M 2$ (b) Wireless broadcast advantage

For an arbitrary network topology, the construction of a routing tree with globally minimum total power expenditure does not have a known algorithm. However, there is a suboptimal solution called the Broadcast Incremental Power (BIP) algorithm that uses a greedy approach to construct a tree. We describe it below.

\subsection{Description of the BIP Algorithm}

Input:given an undirected weighted graph $g(N, A)$, where $N$ :set of nodes, A:set of edges

Initialization: set $\mathrm{T}:=\{\mathrm{S}\}$ where $\mathrm{S}$ is the source node of multicast session.

Set $\mathrm{P}(\mathrm{i}):=0$ for all $1 \leq \mathrm{i} \leq|\mathrm{N}|$ where $\mathrm{P}(\mathrm{i})$ is the transmission power of node $\mathrm{i}$.

Procedure: while $|\mathrm{T}| \neq|\mathrm{N}|$

find an edge $(i, j) \in \mathrm{T} \times(\mathrm{N}-\mathrm{T})$ such that incremental

power $\Delta \mathrm{P}_{\mathrm{ij}}=\mathrm{d}_{\mathrm{ij}}{ }^{\alpha}-\mathrm{P}(\mathrm{i})$ is minimum.

add node $\mathrm{j}$ to $\mathrm{T}$, i.e., $\mathrm{T}:=\mathrm{T} \cup\{\mathrm{j}\}$.

set $\mathrm{P}(\mathrm{i}):=\mathrm{P}(\mathrm{i})+\Delta \mathrm{P}_{\mathrm{ij}}$. 
The BIP algorithm uses the broadcast advantage property while constructing a power efficient tree. As with other heuristic greedy algorithms, this algorithm is not globally optimal in producing a multicast tree with minimum total power expenditure. Currently, there is no known algorithm (except exhaustive search) that leads to a globally optimal solution and is also computationally efficient. Moreover, due to the distributed nature of adhoc networks, the source node may not have global knowledge of network topology in advance without which tree construction is not possible. We present schemes to collect network topology information for three specific cases.

\section{Transmitting Power Information for Power- Efficient Tree Construction}

Since the senders (source/relaying
nodes) do not know the appropriate transmission power level a priori to reach intended receivers, some strategies should be developed to decide the power level based on the feedback from the receivers. The mechanism of exchanging power information is important to maintain the network connectivity. We present solutions to this problem for three different cases depending on the availability of location information or the ability for a receiver to sense the power level. In presenting our solutions, we assume that a transmitter can adjust its power level dynamically.

\subsection{Proposed solution when the location information is available}

It is assumed that location information is provided by using global positioning system (GPS) and each mobile host is equipped with a GPS receiver. The idea of applying GPS to unicast routing was first reported in (Ko and Vaidya, 1998), but their use of GPS was to limit the search space in route discovery process to reduce control overhead, not to determine the transmission power level.
We assume that each node i knows $\left(\mathrm{x}_{\mathrm{i}}, \mathrm{y}_{\mathrm{i}}\right)$ coordinates of itself at every instance of time. By including these coordinates into the header of each packet, and by collecting the positions of multicast member nodes, the sender can easily construct a multicast tree based on the BIP algorithm. Since the coordinate pair can be inserted directly into the IP header, a routing algorithm which resides in the network layer can easily utilize that information. Now the sender node i transmits its beacon or HELLO packet with maximum available power $\mathrm{P}_{\max }$ where the node $\mathrm{i}$ inserts its coordinate. If a receiver node $\mathrm{j}$ is within the transmission range, it can record the coordinate of node $i$ (the backward channel is established). As a response, node $\mathrm{j}$ also transmits with its maximum power by inserting its coordinates in the beacon packet (the forward channel is established). Hence, the amount of minimum transmit power to maintain the link can be easily calculated and the senders can switch to power efficient mode and transmit data packets with minimum required power to preserve battery resource. Because high reliability is usually required for control packets, they have higher priority than data packets, and thus flooding with maximum power $\mathrm{P}_{\max }$ is assumed for the controls packets. If additional information such as velocity is given by GPS, we can constructively utilize this information to optimize several other criteria.

\subsection{When receiver can measure the power level of the received signal}

Let the sender and the receiver be denoted by indices $i$ and $j$. If a receiver can sense the power level of the received signal denoted as $\mathrm{P}_{\mathrm{j}}$, it can record and transmit this value back to the sender $i$. The sender can make use of the knowledge about the attenuation factor $\alpha$ and the maximum power $\mathrm{P}_{\max }$ to compute the required power to node $\mathrm{j}$ as $\mathrm{P}_{\mathrm{ij}}=$ $\mathrm{P}_{\max } / \mathrm{P}_{\mathrm{j}}$. Note that sincethe power level keeps 
changing in a wireless environment depending on the node speed andsurrounding environment due to multipath fading and shadowing, the recorded value should be a statistical average value over a short term interval.

\subsection{Location information is unavailable and receiver cannot measure the power level}

This is the most strict environment in the sense that no information is available which facilitates easy determination of relevant transmit power. One possible solution to determine the power level in this environment is to use an expanding ring search, which is a technique sometimes used in other network applications such as in IGMP. At the network layer, a series of packets are generated with specified power level, $\mathrm{P}_{1}<\mathrm{P}_{2}<\ldots<\mathrm{P}_{\mathrm{L}} \leq \mathrm{P}_{\max }$. Every bit in each control packet is transmitted with the same power level specified in the header. Note that once one of the packets with power $\mathrm{Pi}(1 \leq \mathrm{i} \leq \mathrm{L})$ is captured by the receiver, it can extract the specified power level from the packet header and ignores all the subsequent packets with larger power levels $\mathrm{P}_{\mathrm{i}+1}, \mathrm{P}_{\mathrm{i}+2}, \ldots$, $P_{L}$. This can be achieved by utilizing a broadcast ID and the source address together. Notice that the same broadcast ID should be used for each packet because only one of them will be captured at the receiver and all the others are discarded, i.e., if the source address and the broadcast ID of the packets are the same, only the first captured one with minimum attainable power level is kept.

A guard time between the control packets is desirable to reduce the collision at the receiver and to give enough transition time for a RF transceiver to switch to relevant transmission power level. In this way, a receiver can determine the minimum amount of power level to establish a link from a sender to itself. Although there is a continuous range of assignable power levels, the number of levels should be quantized to a finite number to reduce delay.The choice of individual power level $P_{i}$ and the number of levels will induce inherent performance degradation (accuracy of minimum power level) which is an inevitable result due to the unavailability of local information and direct power measurement.

\section{Proposed Cost Metrics for Network Lifetime Extension}

We define the network lifetime as duration of time until the first node in a network fails due to the battery exhaustion. If all the nodes have identical initial energy levels, the node which dies first will be the one which spends the battery energy at the highest rate. If we want to extend the lifetime of the network, it is critical to incorporate the residual battery energy into route updates. Although the BIP algorithm produces a power efficient multicast routing tree for a single transmission of a packet (which is efficient for a short term period), it does not deal with maximization of the lifetime (which is a long term concept) of a network. Moreover, in a more realistic scenario, the tree structure derived by BIP can not be maintained for a long period of time due to the host mobility, changing environment and dynamic membership change in multicast session, and eventually it has to be updated either periodically or when the network configuration changes. We reformulate the BIP as an optimization problem and propose modified metric for lifetime extension.

\subsection{Reformulation of the BIP algorithm}

Finding a multicast routing tree $\mathrm{T}_{\mathrm{BIP}}$ with BIP algorithm can be reformulated as a corresponding optimization problem as follows:

$$
T_{B I D} \cong \underset{T \subset G(N, A))}{\arg \min } \sum_{(i, j) \in T} \Delta P_{i j}
$$

over all possible trees $T$ that are subgraphs of $\mathrm{G}(\mathrm{N}, \mathrm{A})$ and all edges $(\mathrm{i}, \mathrm{j})$ contained in the tree $\mathrm{T}$. Note that (1) is written as an approximation 
not an equality, because BIP algorithm is not guaranteed to produce a global solution. Similar formulation will be adopted throughout this section for notational simplicity. The exact meaning of it should be interpreted as an algorithmic description explained in section 2.2. The objective here is to minimize the total incremental transmission power defined as a sum of all non-zero incremental powers. The corresponding total transmit power assigned to the tree is:

$$
P_{\text {rootl }}\left(T_{B P}\right)=\sum_{(i, j) \in T_{\text {IIP }}} \Delta P_{i=}=\sum_{i \in N} P(i) .
$$

\subsection{Proposed cost metric for extending network lifetime using Weighted BIP (WBIP)}

We noted that the original BIP algorithm in (1) does not incorporate the residual battery energy into route tree selection. In order to incorporate the residual battery energy into the cost function, we weigh the incremental power $\Delta \mathrm{P}_{\mathrm{ij}}$ while constructing the total weighted cost function $\mathrm{C}_{\mathrm{ij}}$. The weighting function denoted by $\mathrm{W}_{\mathrm{i}}$ for node $\mathrm{i}$ is a time dependent function. The corresponding optimal tree is given by:

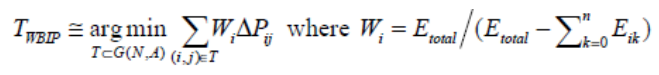

and $E_{\text {total }}$ is the initial battery energy of node $i$, and $E_{i k}$ represents the amount of energy consumed at node $\mathrm{i}$ during the $\mathrm{k}$-th update interval $(\Delta t)$. Therefore, the denominator of $\mathrm{W}_{\mathrm{i}}$ represents the remaining battery energy of node $\mathrm{i}$ at time $\mathrm{t}=\mathrm{n} \Delta \mathrm{t}$. Notice here that the weighting factor $\mathrm{W}_{\mathrm{i}}$ is initially set to unity (therefore, BIP) and as time progresses and as more energy is consumed, $\mathrm{W}_{\mathrm{i}}$ is monotonically increasing (i.e., $\mathrm{W}_{\mathrm{i}} \geq 1$ ). The cost metric $\mathrm{C}_{\mathrm{ij}} \equiv \mathrm{W}_{\mathrm{i}} \Delta \mathrm{P}_{\mathrm{ij}}$ (WBIP)includes both node-based cost and linkbased cost. The battery energy, which is a characteristic of a node, is represented in $\mathrm{W}_{\mathrm{i}}$. The more a node has remaining energy, the less
$\mathrm{W}_{\mathrm{i}}$ is and, therefore, there is a greater chance for this node with large battery capacity to be assigned with a larger transmit power. The reason for $\mathrm{W}_{\mathrm{i}}$ being called node-based cost is that this value is equally weighted to all links to which this node is incident. On the other hand, $\Delta \mathrm{P}_{\mathrm{ij}}$ is a link-based cost because different values are assigned for each link ( $i, j$ ). Although the new cost metric will lead to extension of lifetime of the network, it can often lead to an undesirable oscillatory behavior among paths of the route tree. We first illustrate this behavior and then propose a convex cost function that can reduce the oscillations among routes.

\section{Routing Path Oscillations and Proposed Solution}

In Fig.2, we present the WBIP based routing tree solution at different time instances for 15 nodes in $10 \times 10$ grid with $\alpha=2$. The oscillations of the route paths for different time instances are visually clear if we consider the lower half of the network. The remaining battery level is represented with a shaded rectangle. It can be observed that the battery depletion is evenly distributed among the nodes with this metric. However, the oscillations can have an adverse effect on designing a routing protocol because it can result in out of order packet arrivals. We now present a modification to the cost metric function that will reduce the route oscillations. 
ELK

Asia Pacific Journals
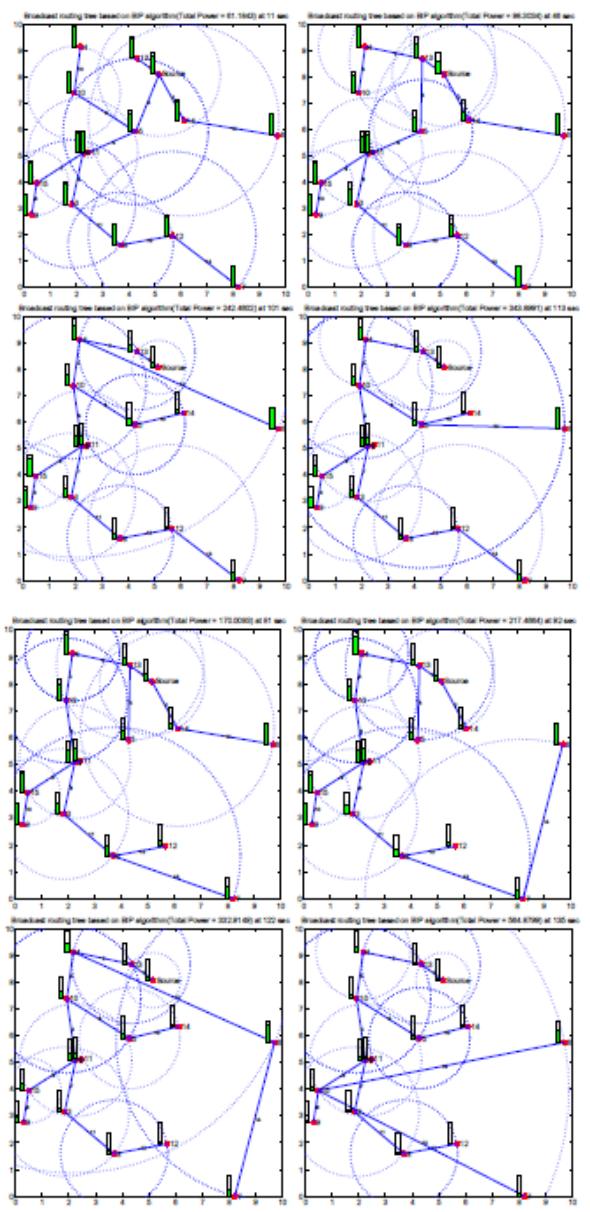

Figure 2: Routing tree oscillation problem for a sample network configuration with 15 nodes

\subsection{Proposed cost metric for simultaneously extending lifetime and reducing route path oscillations (WBIPST):}

Oscillations in routing paths is not a new problem. Some counter measures for oscillation problem are provided in (Khanna and Zinky, 1989). In the case of energyefficient broadcast, the oscillations arise since the WBIP is sensitive to any small change in remaining battery energy level of a node. Our approach uses a statistical measure to reduce oscillations by averaging the costs of links over a time period spanning more than one update intervals (minimizing variance of the link costs is another possible choice). The cost metric
ELK Asia Pacific Journals - Special Issue ISBN: 978-81-930411-5-4

proposed for longer lifetime of the network with stability (WBIPST) is given as:

$\mid T_{\text {WBPST }} \cong \underset{T \subset G(N, A)}{\arg \min } \sum_{(i, j) \in T}{\overline{W_{i}}}^{t} \Delta P_{i j}$ where $\bar{W}_{i}^{t}=\lambda_{t} W_{i}^{t}+\left(1-\lambda_{t}\right){\overline{W_{i}}}_{i}^{t-1}$

and $\lambda_{\mathrm{t}}$ is a time dependent function satisfying 0 $\leq \lambda \mathrm{t} \leq 1$ for all time. This is a modification to (2) with a convex combination of the weighted average of the previous average value and current cost metric value. This cost metric is also a time dependent function and less sensitive to residual battery energy. Note that trees found with these newly proposed metrics (2) and (3)are constructed in the same greedy fashion as BIP and also

not guaranteed to be optimal but better than BIP in terms of lifetime extension.

\section{Simulation Results}

In this section simulations are performed with a simplified network model according to the different metrics BIP, WBIP and WBIPST presented in the previous section. Within a $10 \times 10$ square grid region, network configurations are randomly generated with uniform distribution of nodes and multicast trees are constructed from the source node. Path loss exponents of $\alpha=2,3$ and 4 are separately considered in the simulation. To isolate the effect of each metric, all the generated nodes are assumed to be in the multicast group broadcasting). Initial energy of the battery in each node is assumed to be 1000 units and the broadcast tree is updated at every specified update interval $(\Delta t)$. Constant bit rate $(C B R)$ traffic model is used. The simulation results are for the static network topology without node mobility and no restriction on the 
ELK

Asia Pacific Journals

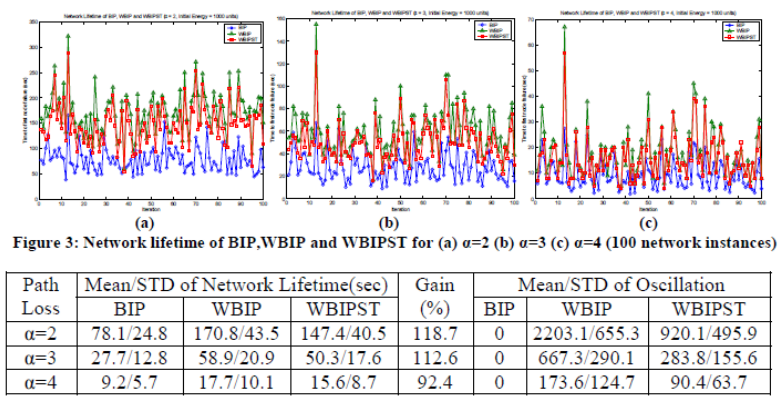

Table 1: Mean and standard deviation of network lifetime and oscillations for 100 network instances: 20 nodes, $\Delta t=1$ (sec) where gain $\equiv($ WBIP-BIP $) /$ WBIP $\times 100 \%$

maximum available transmission power Pmax $=$ $\infty$ is imposed. At every update interval, the amount of energy consumed during the time period is subtracted from the corresponding remaining energy level. Also, the energy consumption by transmission power only is assumed because reception or idle period power is relatively small compared to transmission power.

In Fig.3, the network lifetime is compared for different values of $\alpha=2,3$ and 4 and for $\Delta \mathrm{t}=1$ second with 20 nodes. We used the function $\lambda t=1 / t$ in (3), which is equivalent to recursive formulation of time average, but other functions are also under investigation. In each case, 100 different network topologies are generated and network lifetime was calculated. The same random seeds are used for each metric for valid comparison.

Table 1 summarizes the performance in terms of network lifetime and oscillation count, in which the mean value, standard deviation (STD) and gain (percentage increase in lifetime over BIP) are shown. The number of oscillations is counted as a total sum of the number of

link changes from a previous tree to a current tree until the first node failure. As propagation constant $\alpha$ becomes larger, the lifetime of the network is shortened significantly because the power expenditure is much larger $\left(\mathrm{d}_{\mathrm{ij}}{ }^{4}>\mathrm{d}_{\mathrm{ij}}{ }^{2}\right)$. However, standard deviation becomes smaller as $\alpha$ becomes larger. We can observe that, by using WBIP and WBIPST, network lifetime is roughly prolonged by a factor of two $(\sim 100 \%)$ compared to BIP when $\Delta \mathrm{t}=1$, which is a significant enhancement assuming the given fixed amount of initial battery energy. By using WBIPST, more than half $(58 \%)$ reduction in oscillation from WBIP is achieved at the cost of around $14 \%$ decrease in network lifetime and this is the price paid to reduce route oscillation. We note that oscillation for BIP is identically zero for static network.

The dependence of percentage increase in mean network lifetime of WBIP on the update interval for 100 instances with 20 nodes is shown in Fig.4(a) for $\alpha=2,3$ and 4. For an update interval of 1 second $(\Delta t=1)$, there is about $100 \%$ increase in lifetime which is consistent with the result in Table 1 . It is evident from Fig.4(a) that if tree is updated more frequently, the lifetime is prolonged further. A higher update rate translates to greater control overhead. Therefore the control overhead should be further analyzed so that we can choose a proper update interval in protocol specification. The dependence of lifetime and oscillations on the node density (number of nodes per $10 \times 10$ region) with $\Delta \mathrm{t}=1$ second is presented in Fig.4(b and c). For $\alpha=2$, lifetime increases almost linearly to the node density whereas the increase is more steep for $\alpha=3$ and 4. In summary, our results show that there are essential trade-offs between network lifetime, oscillations, and update interval and therefore proper values should be chosen for protocol design.

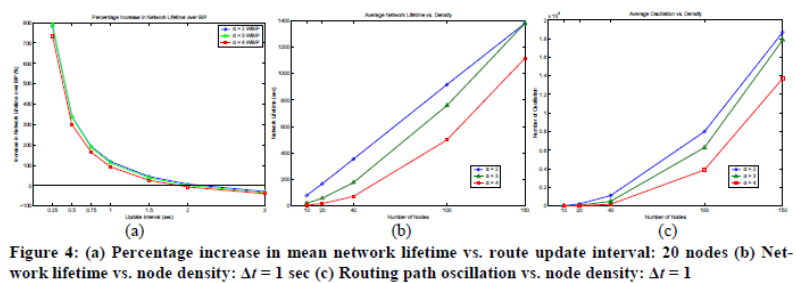

\section{Conclusions}

Our contributions in this paper are, first, we looked at schemes that make the 
ELK

Asia Pacific Journals

construction of a power-efficient tree possible for different scenarios, which will be used for protocol design. Second, we then presented modified cost function that enabled us to extend network lifetime significantly by a factor of two if the tree is updated every second. Finally, we introduced statistical measure in our proposed metric to damp route oscillations and showed that the oscillation can be cut down by half at a small cost of network lifetime.

Current trend of research in multicast routing protocols seems to be leaning toward mesh-based approach mainly because of superior performance of ODMRP. However, our results suggest that the tree-based protocols should also be further pursued because of their energy efficiency. Some of our planned future work involves finding a spanning tree with globally minimum total power, better metrics for lifetime extension and stability, and protocol design of this algorithm to conduct packet level simulation including node mobility.

\section{References}

1.Wieselthier, J.E., Nguyen, G.D. and Ephremides, A. (2000), "On the construction of energy-efficient broadcast and multicast trees in wireless networks," Proc. IEEE INFOCOM 2000, pp. 586-594.

2.Lee, S.-J., Su, W., Hsu, J., Gerla, M. and Bagrodia, R. (2000), "A performance comparison study of ad hoc wireless multicast protocols," INFOCOM 2000, pp. 565-574.

3.Wan, P.-J., Calinescu, G., Li, X.-Y., and Frieder, O. (2001), "Minimum-Energy Broadcast Routing in Static A

Hoc Wireless Networks." Proc. IEEE INFOCOM 2001.
ELK Asia Pacific Journals - Special Issue ISBN: 978-81-930411-5-4

4.Singh, S., Woo, M. and Raghavendra, C.S. (1998), "Power-aware routing in mobile ad hoc networks," Proceedings of MOBICOM, pp. 181-190.

5.Toh, C.-K. (2001), "Maximum battery life routing to support ubiquitous mobile computing in wireless ad hoc

networks," IEEE Communcations Magazine, pp. 138-147.

6.Chang, J.H. and Tassiulas, L. (2000), "Energy conserving routing in wireless ad-hoc networks," INFOCOM '2000. 